\title{
Effects of Electron Impact on Ionization of Short-Lived Isotopes in a Hot Cavity
}

\author{
M. TUREK* \\ Institute of Physics, Maria Curie-Skłodowska University in Lublin \\ pl. M. Curie-Skłodowskiej 1, 20-031 Lublin, Poland
}

\begin{abstract}
A Monte Carlo method based numerical model of hot cavity surface ion source that takes into account radioactive decay and the delay due to the sticking of particles to ionizer surface is considered. The influence of electron impact ionization by thermionic electrons accelerated by the extraction field is investigated. It is shown that the contribution from that process rises with decreasing half-life period and cannot be neglected, especially for substances of small surface ionization coefficient. The changes of relative yields from electron impact and surface processes with the length of the average sticking time are also studied.
\end{abstract}

DOI: 10.12693 /APhysPolA.125.1384

PACS: 07.77.Ka, 07.05.Tp, 34.35.+a

\section{Introduction}

Hot cavity surface ion sources, invented initially for rare-earth isotope separation purposes $[1,2]$ are still used e.g. in nuclear spectroscopy, experimental astrophysics. Due to their advantages like high efficiency, short time nuclides stay in the cavity and high beam purity, they are still developed and optimized [3-11], often evolving into hot cavity resonant ionization laser ion sources (RILIS) $[12,13]$.

A crucial part of the hot cavity ion source is a hollow, elongated, semi-opened cylinder known as ionizer. It is usually heated to high operating temperatures (2000$3500 \mathrm{~K}$ ) either ohmically or using electron beams. The ionizer itself could be an irradiated target in on-line experiments [14]. Otherwise, a transfer line is required [15]. Atoms in the ionizer collide with hot walls and are ionized in a process described by the Saha-Langmuir formula

$$
\alpha=G \exp \left(-\left(V_{\mathrm{i}}-\phi_{\mathrm{e}}\right) / k T\right),
$$

where $V_{\mathrm{i}}$ and $\phi_{\mathrm{e}}$ are the ionization potential of an atom and the work function of the ionizer, respectively, and $G$ is a constant depending on atom/surface composition. The $\alpha$ coefficient is defined as the ratio of the number of ionized $\left(N_{+}\right)$and non-ionized $\left(N_{0}\right)$ atoms leaving the surface. Another often used quantity is the ionization probability $\beta=\alpha /(1+\alpha)$. As a result of multiple collisions of particles with hot walls ionization efficiency may be much greater than that predicted by (1).

Over the years, many analytical models of hot cavity ion source were considered [16-19]. Recently, also a numerical model of ionization in a hot cavity source was developed [20,21] enabling studies of the influence of such factors as ionizer length and geometry, temperature, extraction voltage, atom/surface composition etc.

*e-mail: mturek@kft.umcs.lublin.pl on the ionization efficiency. In its upgraded versions the code was made suitable for spherical ionizers [22] and took into account effects of radioactive decay [23].

The surface ionization (SI) is the main mechanism of ionization occurring in the hot cavity ion sources. However, the production of e.g. multiply charged ions was reported [24], which could not be produced by SI. The authors explained the observed results by existence of ionization mechanism other than SI, namely electron impact ionization (EII) by electrons accelerated by extraction field. Influence of EII on the ionization efficiency of stable nuclides was studied in one of earlier papers [25]. The numerical model was based on the assumption that thermionic electrons emitted from the ionizer may gain enough energy to ionize atoms and significantly contribute to the ion yield. It was shown that for high enough operating temperatures the contribution from EII is non-negligible for small $\beta$ and may be even higher than that from SI.

The aim of that paper is demonstration of the influence of EII on the efficiency of the ion source also in the case of short-lived isotopes, which are of great importance in the field of nuclear spectroscopy. The radioactive decay is modeled using Monte Carlo approach. The estimation of the time spent by an atom inside the ionizer is necessary. Besides the flight time, also the time spent on ionizer walls is taken into account. The estimation of mean free path for EII is given for different ionizer temperatures and EII cross-sections. Changes of EII influence on the ionization efficiency with the radioactive half-life are presented and discussed. The effect of the EII contribution changing with the average sticking time is also under investigation.

\section{Numerical model}

The numerical code solves the equations of motion of particles in the system consisting of a cylindrical ionizer of the length $L$ and the inner radius $r$ and a flat extraction electrode on the potential $-V_{\text {ext }}$ placed at the 
distance $d$ from the ionizer opening. The electrostatic potential distribution is determined by solving the Laplace equation by successive over-relaxation method, similarly as in [26-28]. The simulated system is covered by a numerical grid with $500 \times 100 \times 100$ cells. The cell sizes are: $\Delta x=0.1 \mathrm{~mm}, \Delta y=\Delta z=0.05 \mathrm{~mm}$. The electric field acting on a particle is calculated by the linear interpolation of the values in the nearest grid nodes. The particle trajectory is determined solving classical equation of motion using 4th order Runge-Kutta scheme.

A particle hitting the ionizer wall could be ionized or neutralized with a probability given by the ionization coefficient $\beta$. A particle leaves the surface with the mean velocity corresponding to the ionizer temperature $T$, and the cosine distribution of the initial direction with respect to the normal. The code follows the trajectory of a particle until it passes the opening of the ionizer.

The code takes into account the radioactive decay of primary nuclides. The primary nuclide undergoes the decay after the time $t_{\mathrm{dec}}$, which is determined using the standard Monte Carlo technique

$$
t_{\mathrm{dec}}=-\tau_{1 / 2} \ln (\mathrm{RND}),
$$

where RND is the normal pseudorandom number and $\tau_{1 / 2}$ is the radioactive decay half-life. The code counts the primary and secondary nuclides $\left(N_{p+}\right.$ and $\left.N_{s+}\right)$ as well as neutrals $\left(N_{p 0}\right.$ and $\left.N_{s 0}\right)$. Assuming that the primary nuclide ions are desired kind of particles, the total ion source efficiency is defined as:

$$
\beta_{s}=\frac{N_{p+}}{N_{p+}+N_{s+}+N_{p 0}+N_{s 0}} .
$$

The efficiency decreases with the average time a particle stays inside the ionizer, which is not only the intrinsic delay time (mean time of the particle flight) but also the time spent by a particle on the hot surface. In most cases, the delay in ion production is caused by sticking of particles to the walls. On average, a particle remains on the surface for some time called the sticking time, until it overcomes the potential barrier. The average sticking time on the surface of the temperature $T$ is given by the Frenkel equation [29]:

$$
\tau_{s}=\tau_{0} \exp \left(\frac{\Delta H_{\mathrm{a}}}{k T}\right),
$$

where $\Delta H_{\mathrm{a}}$ is the adsorption enthalpy, $k$ is the Boltzmann constant and $\tau_{0}$ is the inverse Debye frequency. As one can see the average sticking time depends strongly on $T$, but also may vary for different atom/surface combinations. The $\tau_{s}$ is determined using the formula $t_{\text {stick }}=$ $-\tau_{s} \ln \mathrm{RND}$.

The atoms inside the ionizer could be ionized by EII, provided that electrons have kinetic energy larger than ionization energy $E_{\mathrm{i}}$. It is assumed that thermionic electrons from the inner surface of the ionizer near the extraction opening could be accelerated by the extraction field penetrating the ionizer volume (see Fig. 1a) and gain energies larger than ionization threshold. It is expected that only electrons from the cylindrical surface of some length $l$ could have $E>E_{\mathrm{i}}$.
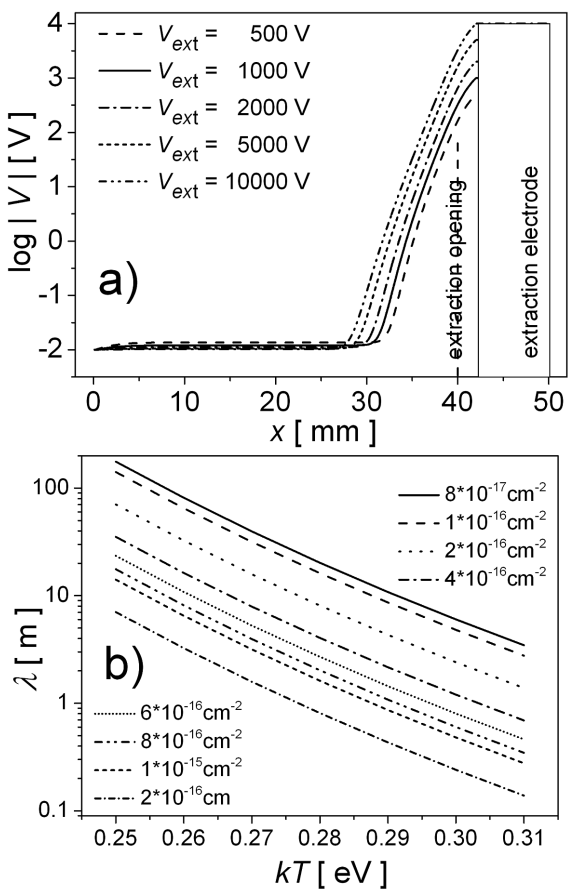

Fig. 1. Potential distribution along the ionizer (a). Mean free path for EII inside the ionizer as a function of its temperature (b).

It is assumed for simplicity that the influence of electron impact of electrons with the energy larger than some cut-off energy $E_{\mathrm{c}}$. The density $n_{\mathrm{e}}$ of electrons with $E>E_{\mathrm{i}}$ may be estimated as in [25]. The mean free path inside the ionizer could be estimated by

$$
\lambda=\left(\sigma n_{\mathrm{e}}\right)^{-1},
$$

where $\sigma$ is the average value of EII cross-section (some experimental data could be found e.g. in [30, 31]). The atom that travels inside the ionizer cavity is ionized by EII after passing the distance

$$
s_{\mathrm{EI}}=-\lambda \ln (\mathrm{RND}) \text {. }
$$

The code tracks the particles until they pass the extraction opening and calculates the total efficiency from the formula (3).

\section{Results}

First step in order to study the influence of EII on the efficiency of the ion source was determination of $\lambda$. Calculations were done for $V_{\text {ext }}=2 \mathrm{kV}, d=2 \mathrm{~mm}$ and $k T$ varying from 0.25 up to $0.31 \mathrm{eV}$. As one can see in Fig. 1b, $\lambda$ decreases with temperature, as well as with $\sigma$, becoming of order of $10 \mathrm{~cm}$ for high enough, but still feasible, temperatures (above $3000 \mathrm{~K}$ ). Changes of EII with $k T$ and $\sigma$ were studied previously [25] for stable isotopes, similar results (not shown here) were obtained for short-lived ones. It is expected that for high $k T$, the contribution from EII for short-lived isotopes is comparable or even larger than that from surface ionization, especially for substances of low $\beta$. 

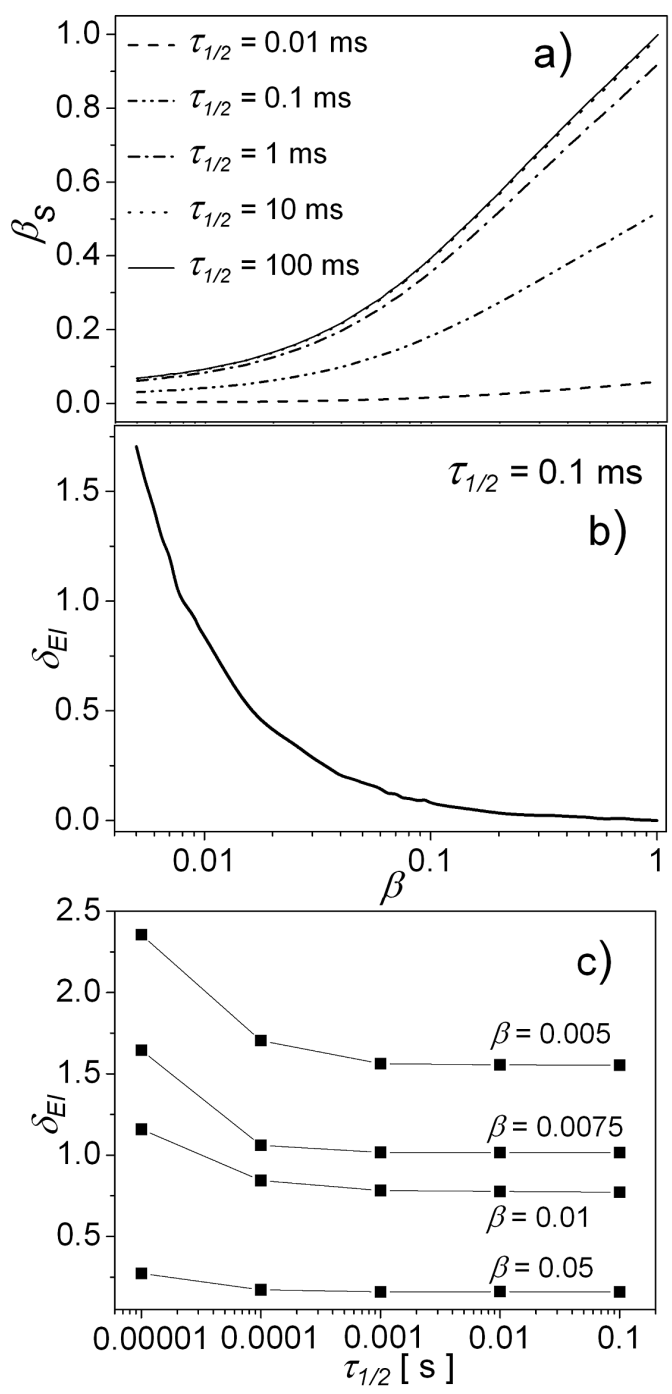

Fig. 2. $\beta_{s}(\beta)$ curves obtained for different $\tau_{1 / 2}$ taking into account both SI and EII processes in the case of $\tau_{s}=0$ (a). The $\delta_{\mathrm{EI}}$ ratio as the function of $\beta$ (b). The $\delta_{\mathrm{EI}}$ ratio as the function of $\tau_{1 / 2}$ (c).

Figure $2 \mathrm{a}$ shows the total ionization efficiency as function of $\beta$ for nuclides of $\tau_{1 / 2}$ changing from $0.01 \mathrm{~ms}$ up to $0.1 \mathrm{~s}$ in the case of the ionizer of the length $L=40 \mathrm{~mm}$ and the radius $r=2.5 \mathrm{~mm}$. The sticking of particles is neglected. Simulations were done employing 300000 of particles of the mass 150 a.m.u. The other parameters were $k T=0.31 \mathrm{eV}$ and $s=1 \times 10^{-15} \mathrm{~cm}^{-2}$. As in the case of pure surface ionization, the efficiency degrades due to the radioactive decay with the decreasing half-life, especially rapidly when $\tau_{1 / 2}$ becomes comparable with the intrinsic delay time $(\approx 0.1 \mathrm{~ms})$.

Figure $2 \mathrm{~b}$ shows the dependence of relative change of $\beta_{s}$ due to EII as the function of $\beta$. It is defined as the ratio:

$$
\delta_{\mathrm{EI}}=\frac{\beta_{s}-\beta_{s}^{0}}{\beta_{s}^{0}},
$$

where $\beta_{s}^{0}$ is the ionization efficiency calculated without taking the electron impact ionization into account. The contribution from EII increases rapidly for small $\beta$ reaching the same magnitude as that from surface ionization for $\beta=0.01$ and becoming almost 1.75 for $\beta=0.005$.

Figure $2 \mathrm{c}$ presents changes of $\delta_{\mathrm{EI}}$ with $\tau_{1 / 2}$, also in the case of $\tau_{s}=0$. It is worth to notice that for small values of $\beta g$ he contribution from electron impact ionization is almost twice larger than that from the surface ionization even for larger $\tau_{1 / 2}$. The $\delta_{\mathrm{EI}}$ ratio increases when $\tau_{1 / 2}$ becomes comparable to the average time a particle stays in the ionizer. This effect is stronger for smaller $\beta$.
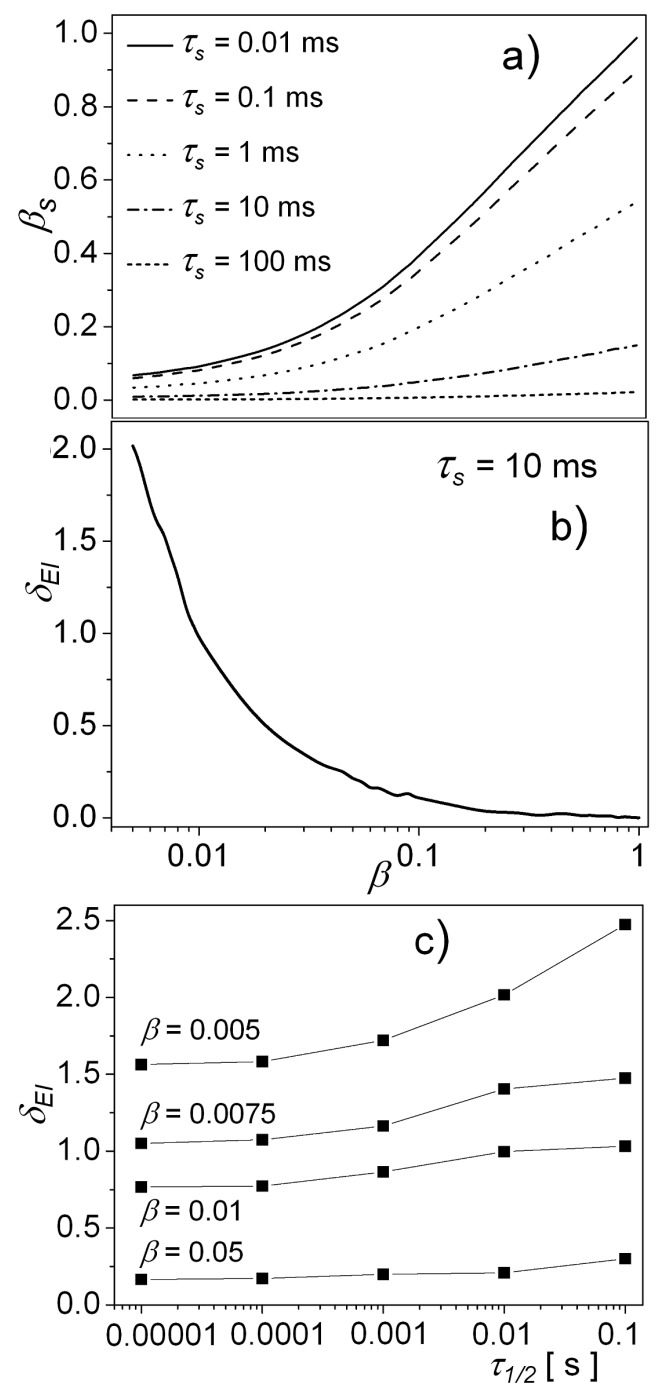

Fig. 3. $\beta_{s}(\beta)$ curves obtained for different $\tau_{s}$ taking into account both SI and EII processes for $\tau_{1 / 2}=$ $0.1 \mathrm{~s}(\mathrm{a})$. The $\delta_{\mathrm{EI}}$ ratio as the function of $\beta(\mathrm{b})$. The $\delta_{\mathrm{EI}}$ ratio as the function of $\tau_{s}$ (c).

The changes of $\delta_{\mathrm{EI}}$ with the average sticking time were also under investigation. Figure 3 a shows $\beta_{s}(\beta)$ curves obtained taking into account both electron impact and surface ionization for $\tau_{1 / 2}=0.1 \mathrm{~s}$ and $\tau_{s}$ changing from $0.01 \mathrm{~ms}$ to $0.1 \mathrm{~s}$. The degradation of $\beta_{s}$ is observed as $\tau_{s}$ grows due to the multiple collisions with walls. The 
mean time of staying in the ionizer exceeds $\tau_{1 / 2}$ even for $\tau_{s}=1 \mathrm{~ms}$. As in the case of $\tau_{s}=0$, one can observe that the contribution of EII is important for small $\beta$, reaching even $200 \%$ of that from the surface process (Fig. 3b). Figure $3 c$ shows changes of $\delta_{\mathrm{EI}}$ ratio with $\tau_{s}$. Despite the fact that $\beta_{s}$ falls fast with $\tau_{s}, \delta_{\mathrm{EI}}$ rises, being of order of 1 for $\beta=0.01$. For small $\beta$ the electron impact contribution is non-negligible in the whole considered range of $\tau_{s}$ reaching even $250 \%$ of the surface ionization yield.

\section{Conclusions}

The realistic model of ionization in the hot cavity surface ion source was considered, including such effects as radioactive decay, sticking of atoms to hot walls and the influence of electron impact ionization. It was found that EII influence rises with decrease of $\beta$ and could not be neglected for substances of $\beta<0.01$. The contribution from that process could be larger (even twice) than the yield from surface ionization and it rises with decrease of $\tau_{1 / 2}$. The changes of relative yield from EII with $\tau_{s}$ were studied. It was found that it rises significantly with $\tau_{s}$. This effect is stronger for substances of low $\beta$.

The effect of electron impact ionization should be taken into account for substances of low $\beta$, especially for short-lived isotopes and in the case of long sticking times, as it may change the model predictions by several hundreds percent.

\section{References}

[1] G.J. Beyer, E. Herrmann, A. Piotrowski, V.I. Raiko, H. Tyroff, Nucl. Instrum. Methods 96, 437 (1971).

[2] P.G. Johnson, A. Bolson, C.M. Henderson, Nucl. Instrum. Methods 83, 106 (1973).

[3] V.N. Panteleev, Rev. Sci. Instrum. 75, 1602 (2004).

[4] G.D. Alton, Y. Liu, D.W. Stracener, Rev. Sci. Instrum. 77, 03A711 (2006).

[5] C. Jost, H.K. Carter, R.E. Goans, B.O. Griffith, R. Katakam, K.-L. Kratz, C.A. Reed, E.H. Spejewski, T. Stora, D.W. Stracener, AIP Conf. Proc. 1099, 750 (2009).

[6] M. Manzolaro, G. Meneghetti, A. Andrighetto, Nucl. Instrum. Methods Phys. Res. A 623, 1061 (2010).

[7] Y. Otokawa, A. Osa, T.K. Sato, M. Matsuda, S. Ichikawa, S.C. Jeong, Rev. Sci. Instrum. 81, 02A902 (2010).

[8] J. Montano, J. Vasquez, A. Andrighetto, G. Bassato, L. Calabretta, M. Poggi, G. Prete, Nucl. Instrum. Methods Phys. Res. A 648, 238 (2011).

[9] T. Stora, Nucl. Instrum. Methods Phys. Res. B 317, 402 (2013).

[10] M. Manzolaro, A. Andrighetto, G. Meneghetti, M. Rossignoli, S. Corradetti, L. Biasetto, D. Scarpa, A. Monetti, S. Carturan, G. Maggioni, Nucl. Instrum. Methods Phys. Res. B 317, 446 (2013).

[11] Y. Blumenfeld, T. Nilsson, P. Van Duppen, Phys. Scr. T 152, 014023 (2013).
[12] Y. Liu, C.U. Jost, A.J. Mendez II, D.W. Stracener, C.L. Williams, C.J. Gross, R.K. Grzywacz, M. Madurga, K. Miernik, D. Miller, S. Padgett, S.V. Paulauskas, K.P. Rykaczewski, M. WolinskaCichocka, Nucl. Instrum. Methods Phys. Res. B 298, 5 (2013).

[13] V.N. Fedosseev, L.-E. Berg, D.V. Fedorov, D. Fink, O.J. Launila, R. Losito, B.A. Marsh, R.E. Rossel, S. Rothe, M.D. Seliverstov, A.M. Sjödin, K.D.A. Wendt, Rev. Sci. Instrum. 83, $02 \mathrm{~A} 903$ (2012).

[14] V.G. Kalinnikov, K.Ya. Gromov, M. Janicki, Yu.V. Yushkevich, A.W. Potempa, V.G. Egorov, V.A. Bystrov, N.Yu. Kotovsky, S.V. Evtisov, Nucl. Instrum. Methods Phys. Res. B 70, 62 (1992).

[15] G.D. Alton, Y. Zhang, Nucl. Instrum. Methods Phys. Res. A 539, 540 (2005).

[16] A. Latuszyński, V.I. Raiko, Nucl. Instrum. Methods 125, 61 (1975).

[17] V.P. Afanas'ev, V.A. Obukhov, V.I. Raiko, Nucl. Instrum. Methods 145, 533 (1977).

[18] R. Kirchner, Nucl. Instrum. Methods Phys. Res. A 292, 203 (1990).

[19] A. Latuszyński, K. Pyszniak, A. Droździel, M. Turek, D. Mączka, J. Meldizon, Vacuum 81, 1150 (2007).

[20] M. Turek, K. Pyszniak, A. Drozdziel, J. Sielanko, Vacuum 82, 1103 (2008).

[21] M. Turek, A. Drozdziel, K. Pyszniak, D. Maczka, B. Slowinski, Rev. Sci. Instrum. 83, 023303 (2012).

[22] M. Turek, Acta Phys. Pol. A 120, 188 (2011).

[23] M. Turek, Acta Phys. Pol. A 123, 847 (2013).

[24] D.M. Wayne, W. Hang, D.K. McDaniel, R.E. Fields, E. Rios, V. Majidi, Int. J. Mass Spectrom. 216, 41 (2002).

[25] M. Turek, K. Pyszniak, A. Droździel, Vaccum 83. S260 (2009)

[26] A. Pyszniak, A. Droździel, M. Turek, A. Latuszyński, D. Maczka, J. Sielanko, Yu.A. Vaganov, Yu.V. Yushkevich, Instrum. Exp. Techniq. 50, 552 (2007).

[27] M. Turek, J. Sielanko, Vacuum 83, S256 (2009).

[28] M. Turek, A. Drozdziel, K. Pyszniak, S. Prucnal, J. Żuk, Przeglad Elektrotechniczny 7, 193 (2010) (in Polish).

[29] A. Zangwill, Physics at Surfaces, Cambridge University Press, Cambridge 1988.

[30] S. Yagi, T. Nagata, J. Phys. Soc. Jpn. 70, 2559 (2001).

[31] Y.K. Kim, P.M. Stone, Phys. Rev. A 64, 052707 (2001). 